\title{
When do open-loop strategies for combustion control work?
}

\author{
R. Prasanth, A.M. Annaswamy ${ }^{\dagger}$ J.P. Hathout ${ }^{\ddagger}$ and A.F. Ghoniem ${ }^{\dagger}$
}

\begin{abstract}
This paper addresses open-loop control strategies that involve a low frequency modulation of the fuel injection for suppression of pressure oscillations in combustion systems. Such a strategy has been employed in a number of recent investigations including [1]-[5]. Using a physically based model, analytical explanations for the effectiveness of such a low pulsing strategy are provided in this paper. Conditions are derived on the frequency and the duty-cycle used in the control strategy that lead to stabilization in the combustor, under ideal and nonideal switching strategies, and in the presence of hysteresis.
\end{abstract}

\section{Introduction}

An important performance requirement of lean premixed combustion systems is stability over the entire operating range of fuel/air-flow. Unstable pressure oscillations are undesirable as they impact the component life-time and the overall turbine in general. Avoiding unstable operating conditions by increasing the equivalence ratio negatively affects other desirable performance specifications such as low NOx and high efficiency.

Combustion instability has been observed in systems such as afterburners, gas turbines, waste incinerators. Practical solutions to avoid it include changes in the acoustic parameters and/or the combustion stabilization mechanisms as well as the use of dampers. Lack of understanding of the underlying mechanisms, models that can predict the instability characteristics as a function of the system geometry, design and operating conditions, make it difficult to design stable systems without the use of numerous iterations.

Active control technologies have been explored over the past decade in an effort to combat the unstable dynamics without requiring major hardware redesigns, limiting the operability regime of the engine, or shifting the operating condition away from the state of lowest emission in lean systems or highest power density in near stoichiometric systems. Five different methods have been adopted for active control: (i) Phase-shift control, which consists of filtering, phase-shifting, and feeding back the pressure signal [6]-[13]

\footnotetext{
${ }^{*}$ Research Engineer, Scientific Systems Inc., Woburn, MA 01801.

${ }^{\dagger}$ Department of Mechanical Engineering, MIT, Cambridge, MA 02139

${ }^{\ddagger}$ Senior Systems Engineer, Robert Bosch Corp. Research and Technology Center, Pittsburgh, PA 15212.
} 
(ii) model-based control, which consists of developing a physically based model of the combustion dynamics and have been demonstrated to yield an optimal stabilizing response under certain conditions, [14]-[17], (iii) observer-based control [18], which have been shown to result in satisfactory pressure suppression using an on-line determination of an observer and a phase-shift controller based on the observer parameters, (iv) control based on system-identification based modeling [19, 20] and (v) open-loop control strategies [1, 2],[21]-[5] where the mass-flow rate of a fuel injector (and therefore the equivalence ratio) is switched between two different values at a significantly slower frequency than the resonant frequency. This paper concerns analytical discussions of the last method listed above.

In $[1,2,21]$ a low frequency pulsing strategy was used to stabilize the pressure oscillations, with no feedback. In [3], a similar low-frequency modulation was used. In [4, 5], the presence of hysteresis in the combustor was used to design a low-frequency pulsing strategy to suppress the pressure oscillations. In [1], a $20 \mathrm{~kW}$ gas turbine combustor was stabilized, with a resonant frequency of $300 \mathrm{~Hz}$ and an equivalence ratio of 0.85 , using a fuel-injector oscillated at $50 \mathrm{~Hz}$ and at duty cycles between 0.36 and 0.5 . It was also observed that for larger duty-cycles (than 0.6), the same injector led to oscillations of a higher amplitude than without control. In [2], a similar rig was studied and the same switching strategy was used, but with different switching frequency and duty-cycle at different operating conditions. It was shown that the 300 $\mathrm{Hz}$ oscillations are reduced significantly by switching between equivalence ratios of 0.65 and 0.75 , and an air-flow rate of $20.7 \mathrm{~g} / \mathrm{s}$, for all switching frequencies less than $20 \mathrm{~Hz}$, and duty-cycles close to 0.5 . The hypothesis proposed in [2] for why stabilization occurs was that these two values of the equivalence ratio correspond to "stable" operating points and therefore stabilization occurs by switching between these two "stable" values though the intermediate value of $\phi=0.7$ corresponds to an "unstable" operating point. In [21] practical considerations of deployment of fuel-injectors, in terms of their number as well as location that generate such "stable" operating points, were discussed.

The question that arises is, why does such an open-loop control strategy work? What are the limits of operation of such a methodology? Given that low-frequency pulsing is an attractive method since it circumvents the constraints due to the currently available bandwidth of available fuel-injectors, what are the guidelines for fuel-injector pulsing strategy as well as fuel-injector design in terms of number and location for optimal pressure suppression? If hysteresis is indeed present in a combustor, what are the guidelines for designing a switching strategy? We will attempt to answer these questions in this paper. 


\section{An Analytical Model}

In this section, we model the combustion dynamics by deriving models of heat release, acoustics and inhomogeneity dynamics, and the coupling dynamics.

\subsection{Heat Release Dynamics}

At high Damkohler numbers and weak to moderate turbulence intensity, turbulent combustion can be modeled using wrinkled laminar flames $[22,23]$. Incorporating the effects of perturbations in the equivalence ratio, the flame surface can be described by a single-valued function $\xi(r, t)$ and the total heat release, $Q$, is proportional to the integral of this surface over an anchoring ring. These relations are given by

$$
\begin{aligned}
\frac{\partial \xi}{\partial t} & =u-v \frac{\partial \xi}{\partial r}-S_{u}(\phi) \sqrt{\left(\frac{\partial \xi}{\partial r}\right)^{2}+1} \\
Q & =\kappa(\phi) \int_{0}^{R} \sqrt{1+\left(\frac{\partial \xi}{\partial r}\right)^{2}} d r
\end{aligned}
$$

with $S_{u}$, the burning velocity, and $\Delta h_{r}$, the heat of reaction being a functions of $\phi, \kappa(\phi)=2 \pi \rho_{u} S_{u}(\phi) \Delta h_{r}(\phi)$, and $\rho_{u}$ is the density of the unburnt mixture. To derive a linear model, the effects of perturbations in both $u$ and $\phi$ will be considered.

Assuming negligible velocity component in the radial direction, and linearizing around nominal values $\bar{u}, \bar{S}_{u}$, and $\bar{\xi}(r)$, denoting $\overline{(.)}$ and (.)' as steady and perturbation, respectively, we can derive the following linear model [24]:

$$
\dot{Q}^{\prime}=d_{0} u^{\prime}+d_{1}\left(u_{\tau_{f}}^{\prime}(t)\right)+d_{2}\left(\phi_{\tau_{f}}^{\prime}(t)\right)+d_{3} \phi^{\prime}+d_{\phi} \dot{\phi}^{\prime}
$$

where

$$
x_{\tau}(t) \triangleq \int_{t-\tau}^{t} x(\zeta) d \zeta, \quad \tau_{f}=\frac{R}{\bar{S}_{u}}
$$

the coefficients $d_{i}$ depend on $S_{u}$ and $\phi$, and $\tau_{f}$ represents the characteristic propagation delay of the flame surface into the reactants flow.

\subsection{Acoustics}

Combustors exhibit instabilities over a range of frequencies including the Helmholtz mode, the longitudinal, and transverse modes. These represent the acoustic component of the instability, or the host oscillator. The 
equations governing the first two are given by

$$
\begin{aligned}
\frac{d^{2} p^{\prime}}{d t^{2}}+2 \zeta \omega \frac{d p^{\prime}}{d t}+\omega^{2} p^{\prime} & =\frac{\gamma-1}{V} \dot{q}^{\prime}(x, t), \\
\frac{\partial^{2} p^{\prime}}{\partial t^{2}}-\bar{c}^{2} \frac{\partial^{2} p^{\prime}}{\partial x^{2}} & =(\gamma-1) \dot{q}^{\prime}(x, t),
\end{aligned}
$$

In the above, $\omega=\sqrt{\frac{c_{i}^{2} A_{i}}{L_{i} V}+\frac{c_{e}^{2} A_{e}}{L_{e} V}}$ is the effective Helmholtz frequency [25] associated with a combustor connected to ducts. The passive damping due to different dissipation sources, e.g. heat-loss and friction, is accounted for in the natural damping ratio, $\zeta$, and $\bar{c}$ is the mean speed of sound. The same approach can be used for transverse modes as well (for example, screech modes in rockets [26]). In what follows, we assume that flames are localized close to the anchoring plane, so that $q^{\prime}(x, t)=q^{\prime}(t) \delta\left(x-x_{f}\right)$.

Using an expansion in basis functions for both Eqs. (4) and (5) as

$$
p^{\prime}(x, t)=\bar{p} \sum_{i=0}^{n} \psi_{i}(x) \eta_{i}(t),
$$

where $\psi_{0}$ is a constant, since it corresponds to the spatial variation in the bulk-mode, $\psi_{i}(x)=\sin \left(k_{i} x+\phi_{i 0}\right)$, $i=1, \ldots, n$, and $k_{i}$ and $\phi_{i 0}$ determined from the boundary conditions. Performing a weighted spatial averaging, the modal amplitudes can be shown to follow [14]:

$$
\ddot{\eta}_{i}+2 \zeta \omega_{i} \dot{\eta}_{i}+\omega_{i}^{2} \eta_{i}=\sum_{i=1}^{n} \widetilde{b}_{i} \dot{q}_{f}^{\prime}
$$

where $\widetilde{b}_{0}=(\gamma-1) / V, \widetilde{b}_{i}=\gamma a_{o} \psi_{i}\left(x_{f}\right) / E$ for $i=1, \ldots, n, E=\int_{0}^{L} \psi_{i}^{2}(x) d x, \gamma$ is the specific ratio, $a_{o}=\frac{\gamma-1}{\gamma \bar{p}}, \zeta$ represents the passive damping ratio in the combustor ${ }^{1}, L$ is its length, $\omega_{0}^{2}=\sqrt{\frac{A_{n}}{L_{n} V}}, V$ is the volume of the combustor, $A_{n}$ and $L_{n}$ are the cross-sectional area and length of the inlet/outlet neck connected to the combustor. and $\omega_{i}=k_{i} \bar{c}, i=1, \ldots, n$. Typically, $\omega_{0}<<\omega_{i}$, for $i=1, \ldots, n$.

\subsection{Coupling Dynamics}

In the case when a Helmholtz-type resonance is triggered in the combustor, the acoustic velocity is very small and the possible coupling between heat release fluctuations and acoustics is through the pressure which in turn produces perturbations in the equivalence ratio via feedline dynamics [27]. In particular, if either the air or fuel-flow feeds is choked and the other feed is unchoked, $\phi$ can fluctuate. The instantaneous equivalence ratio $\phi_{s}^{\prime}$, when linearized, can be shown to satisfy the relation

$$
\phi_{s}^{\prime}=-\frac{\bar{\phi}}{\bar{u}} u_{s}^{\prime}
$$

\footnotetext{
${ }^{1}$ Dissipation in a combustor can be caused by heat losses in the flame zone and friction due to viscous effects.
} 
In addition, there is a convective delay $\tau_{c}$ due to transport lag from the supply to the burning plane of the flame, and hence,

$$
\phi^{\prime}=\phi_{s}^{\prime}\left(t-\tau_{c}\right)
$$

where $\tau_{c}=L / \bar{u}$, and $L$ is the distance between the supply and the burning plane.

The equivalence ratio perturbations, in turn, can be related to the pressure perturbations in the combustor by considering the momentum conservation in the inlet duct,

$$
\frac{\partial u_{i}^{\prime}}{\partial t}+\frac{1}{\rho_{i}} \frac{\partial p_{i}}{\partial x}=0
$$

where $u_{i}$ and $p_{i}$ denote the velocity and the pressure at the inlet duct, respectively. When the dominant acoustic modes are longitudinal, both perturbations in $u$ and $\phi$ can induce instability. The coupling between $u$ and $p$ can be determined using the energy conservation equation as

$$
\frac{\partial p^{\prime}}{\partial t}+\gamma \bar{p} \frac{\partial u^{\prime}}{\partial x}=(\gamma-1) q^{\prime}
$$

\subsection{Overall Model}

Combining the acoustics, heat-release, and convective lag effects, we obtain the following equations:

$$
\ddot{\eta}_{i}+2 \zeta \omega_{i} \dot{\eta}_{i}+\omega_{i}^{2} \eta_{i}=\widetilde{b}_{i}\left[d_{0} u^{\prime}+d_{1}\left(u_{\tau_{f}}^{\prime}(t)\right)+d_{2}\left(\phi_{\tau_{f}}^{\prime}(t)\right)+d_{3} \phi_{s}^{\prime}\left(t-\tau_{c}\right)+d_{\phi} \dot{\phi}_{s}^{\prime}\left(t-\tau_{c}\right)\right]
$$

This indicates that two different coupling mechanisms are possible excitations for the acoustics, one resulting from the velocity perturbations $u^{\prime}$ and the other from equivalence ratio perturbations $\phi^{\prime}$. Eq. (12) also indicates that two different time-delays, $\tau_{f}$ and $\tau_{c}$ can induce these excitations, one arising from propagation effects, and the other from convection.

The complete combustion dynamics is therefore determined by (12) and the coupling relations given by (9), (10) and (11). For ease of exposition we assume that only one acoustic mode is present, and set $\eta_{i}=\eta$. If the variations are mainly in $u^{\prime}$, then Eqs. (12) and (11) can be combined to obtain the relation

$$
\ddot{\eta}+2 \zeta_{0} \omega_{0} \dot{\eta}+\omega_{0}^{2} \eta-\gamma_{2} \eta\left(t-\tau_{f}\right)=0
$$

where

$$
2 \zeta_{0} \omega_{0}=2 \zeta \omega-\gamma_{1}, \quad \omega_{0}^{2}=\omega^{2}+\gamma_{2}, \quad \gamma_{1}=\frac{R}{\bar{S}_{u}} \gamma_{2}, \quad \gamma_{2}=\bar{\kappa} \bar{S}_{u} \frac{\bar{p} a_{o}}{E k^{2}}\left(\frac{d \psi}{d x} \psi(x)\right)_{x_{f}}, \quad \tau_{f}=\frac{R}{\bar{S}_{u}}
$$

and if they are due to $\phi^{\prime}$, then Eqs. (12), (10), and (9) can be combined to obtain

$$
\dddot{\eta}+2 \zeta \omega \ddot{\eta}+\omega_{i}^{2} \dot{\eta}-\beta_{1} \dot{\eta}\left(t-\tau_{c}\right)+\beta_{2} \eta\left(t-\tau_{c}\right)-\beta_{3} \eta_{f c}(t)=0, \quad \eta_{f c}=\int_{t-\tau_{f}-\tau_{c}}^{t-\tau_{f}} \eta(\zeta) d \zeta
$$


where

$$
\begin{aligned}
& \beta_{1}=2 \pi \widetilde{b} \frac{\bar{\phi}}{\bar{u}} \widetilde{c} k^{2}\left(\left.\bar{S}_{u} \frac{d \Delta h_{r}}{d \phi}\right|_{\bar{\phi}}+\left.\Delta \bar{h}_{r} \frac{d S_{u}}{d \phi}\right|_{\bar{\phi}}\right)\left(\int_{0}^{R} \bar{\xi} d r\right), \beta_{2}=2 \pi \widetilde{b} \frac{\bar{\phi}}{\bar{u}} \widetilde{c} k^{2} \Delta \bar{h}_{r} \bar{S}_{u} \frac{d \bar{S}_{u}}{d \phi} \bar{\xi}(0) \\
& \beta_{3}=-\left.2 \pi \Delta \bar{h}_{r} \widetilde{b} \frac{\bar{\phi}}{\bar{u}} \widetilde{c} \gamma k^{2} \bar{S}_{u}^{2} \frac{d \bar{S}_{u}}{d \phi} \frac{d \bar{\xi}}{d r}\right|_{0}, \quad \tau_{c}=\frac{L}{\bar{u}}
\end{aligned}
$$

At the acoustic frequency, the impact of the fifth and sixth term on the left-hand-side of Eq. (15) are typically smaller. Also $\tau_{f}$ is small compared to $\tau_{c}$ in many cases. Hence a simplified version of (15) can be analyzed in the form

$$
\ddot{\eta}+2 \zeta \omega \dot{\eta}+\omega^{2} \eta-\beta_{1} \eta\left(t-\tau_{c}\right)=0
$$

It is interesting to note that the structure of (16) is identical to that of (13) with the differences only due to the parameters. This implies that time-delay effects are present both in the presence of $u^{\prime}$ - and $\phi^{\prime}$-perturbations,

with the distinction that with $u^{\prime}$, the time-delay is due to $\tau_{f}$, which is due to flame propagation, and with $\phi^{\prime}$, the delay is due to $\tau_{c}$, a convection effect. The other distinction is in the damping effect; in the former, if $\gamma_{1}$ is positive, even in the absence of any time-delay, instabilities can be present. In the latter, on the other hand, instability is only due to the time-delay $\tau_{c}$; the damping effect is stabilizing.

\subsection{Discussion}

The models in (16) and (13) were discussed at length in [24], and were shown to exhibit intervals of alternating stable and unstable behavior as the time-delay increased. These models were also shown to predict the instability behavior of a number of experimental rigs. In particular, the model in (16) was shown to alternate between stability and instability as the convective lag $\tau_{c}$ increased, which was similar to the properties of the rig considered in [1]. Similarly, the model in (13) appears to match the stability map in Figure 6 in [2] which shows the stability bands.

A few comments about the various parameters in (13) and (16) are in order. We discuss the parameters in (13) first. The dependence of $\gamma_{i}$ 's on $\phi$ is straight-forward, due to the changes of $\Delta h_{r}$ and $S_{u}$ with $\phi$. In general, for values of $\phi \leq 1, \gamma_{0}$ and $\gamma_{1}$ increase with $\phi$. Similarly, $\tau_{f}$ increases with a decreasing $\bar{\phi}$ due to its dependence on $S_{u}$ for values away from the LBO limit and unity. The dependence of $\omega$ on $\phi$ is indirect, and occurs through the temperature which increases as $\phi$ increases and hence increases the speed of sound and therefore the frequency. The quantity $\zeta_{0}$ depends on the flame position with respect to the acoustic mode. For example, a quarter wave acoustic mode implies that $\gamma_{1}$ is negative and therefore $\zeta_{0}$ is positive, and hence, leads to a stable system for small values of $\tau_{f}$, and destabilized as $\tau_{f}$ increases. 
The paremeters in (16) are also dependent on $\bar{\phi}$ but to a smaller extent. For example, changes in $\tau_{c}$ are more likely to occur with changes in the mean velocity $\bar{u}$ rather than $\bar{\phi}$. Even though the parameter $\beta_{1}$ changes with $S_{u}$ and $\Delta h_{r}$ as $\phi$ increases, since it occurs as a linear coefficient in (16), its changes do not produce drastic differences in the stability characteristics of (16). The damping coefficient is unchanged with flame position, while the frequency dependence with $\phi$ is the same as in (13) due to temperature changes.

\section{Stabilization using the Open-loop Control Strategy}

In this section, we address the problem of stabilization using an open-loop control strategy that consists of switching between two values of equivalence ratio. In particular, we will focus on the strategies used in $[1,2]$ which consists of changing the equivalence ratio between two constant values at a frequency that is significantly lower than the resonant frequency. The discussions in the previous section indicate that while the specific dependence of the individual parameters may be different, the general class of models that describe the combustion instability are of the form of

$$
\ddot{\eta}+2 \zeta_{0} \omega \dot{\eta}+\left(\omega^{2}-k_{1}\right) \eta+k_{2} \eta(t-\tau)=0
$$

with the exact variations in $\zeta_{0}, \omega, k_{1}, k_{2}$ and $\tau$ depending on whether the instability is due to $u^{\prime}$ or $\phi^{\prime}$. Using the model in (17), we derive the conditions under which the switching strategy can stabilize the combustion dynamics. Design procedures for meeting these stabilization conditions are also discussed.

\subsection{Ideal Switching}

The control strategy in $[1,2]$ can be described as follows: Starting from $t_{0}=0$, define $t_{i}=t_{i-1}+T_{m}$ for $i \geq 1$ and modulate equivalence ratio according to:

$$
\phi(t)= \begin{cases}\phi_{1} & \text { for } t_{i-1} \leq t<t_{i-1}+W_{p} T_{m} \\ \phi_{2} & \text { for } t_{i-1}+W_{p} T_{m} \leq t<t_{i}\end{cases}
$$

where $0 \leq W_{p} \leq 1$ is the pulse width and $T_{m}$ is the pulse period. The parameters $\left(\omega, \zeta_{0}, k_{1}, k_{2}, \tau\right)$ of the model (17) are functions of $\phi$ and, under this ideal switching strategy, the system (17) becomes a timevarying time-delay system. Our objective is to analyze the stability of this system and derive conditions from which the design variables $\left(\phi_{1}, \phi_{2}, W_{p}, T_{m}\right)$ can be computed readily.

Let us rewrite (17) in the following way:

$$
\ddot{\eta}+2 \zeta_{\phi} \omega_{\phi} \dot{\eta}+\left(\omega_{\phi}^{2}-k_{1 \phi}\right) \eta+\alpha_{1 \phi} \eta\left(t-\tau_{\phi_{1}}\right)+\alpha_{2 \phi} \eta\left(t-\tau_{\phi_{2}}\right)=0
$$


where subscripts have been introduced to show dependence of the parameters on equivalence ratio $\phi$. We have also brought in two new functions $\alpha_{1 \phi}$ and $\alpha_{2 \phi}$ that switch according to:

$$
\alpha_{k \phi}= \begin{cases}k_{2 \phi_{k}} & \text { if } \phi=\phi_{k} \\ 0 & \text { otherwise }\end{cases}
$$

The procedure outlined below establishes, in a rigorous manner, that the system in (19) can be stabilized using a large enough $T_{m}$ provided there are two operating points with equivalence ratios $\phi_{1}$ and $\phi_{2}$ on either side of the desired unstable operating point with at least one of them being stable. Once this is established, the question then remains is to determine the conditions under which such a stability pattern exists in a given rig. In particular, we note that the stability of the system in (19) is dependent on the parameter values of $f(\phi)$, where

$$
f(\phi)=\left(\omega_{\phi}, \zeta_{\phi}, k_{1 \phi}, \alpha_{1 \phi}, \alpha_{2 \phi}, \tau_{\phi}\right)
$$

which obviously changes with $\phi$. This dependence is discussed in Section 6 .

\subsubsection{Stability Conditions Under Ideal Switching}

It is clear that (19) describes (17) under the control strategy (18). When $\phi$ is a constant, the system (19) can be written in feedback interconnection form as

$$
\begin{aligned}
& \hat{y}=\frac{s+1}{\left(s^{2}+2 \zeta_{\phi} \omega_{\phi} s+\left(\omega_{\phi}^{2}-k_{1 \phi}\right)\right)} u=(s+1) G_{\phi}(s) u
\end{aligned}
$$

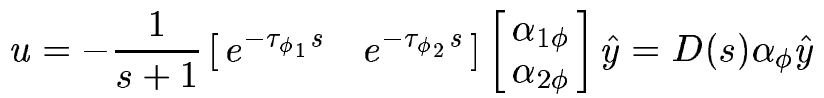

$$
\begin{aligned}
& \eta=y
\end{aligned}
$$

where the infinite dimensional component $D$ is time-invariant. This feedback interconnection is shown on the left of Figure 1. The block diagram shows that we have simply added a stable pole and a canceling minimum phase zero in order to generate a finite dimensional uniform approximation.

Now, given any $\gamma>0$, there exists a finite dimensional system $P$ with a rational transfer function of degree say $2 n$ such that:

$$
P+\Delta=D(s)=\frac{1}{s+1}\left[e^{-\tau_{\phi_{1}} s} e^{-\tau_{\phi_{2}} s}\right]
$$

where the approximation error $\Delta$ satisfies

$$
|\Delta(j \omega)| \leq \frac{1}{\gamma}
$$

for all frequency $\omega$ including $\infty$. Similarly, given any order $n$, we can compute a system $P$ of order $n$ and a number $\gamma>0$ such that the approximation error is smaller in magnitude than $1 / \gamma$ at every frequency. 

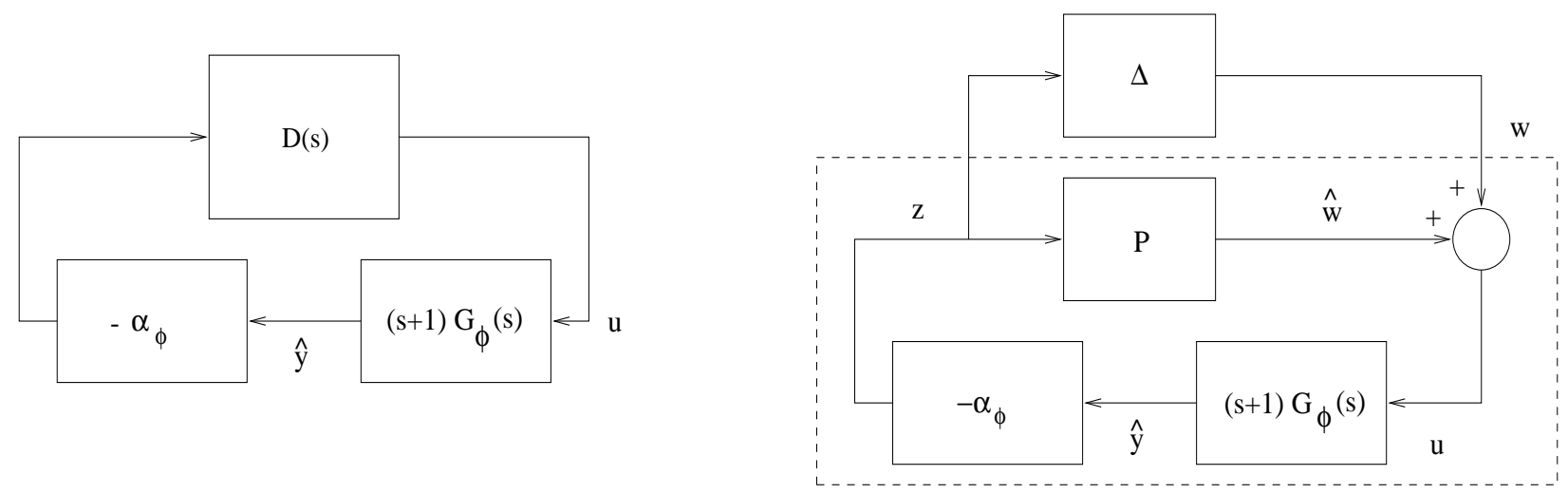

Figure 1: Block diagram for approximation procedure

Moreover, as $n$ goes to infinity, the approximation error goes to zero uniformly. This additive representation of the infinite dimensional part of (19) is shown on the right hand side of Figure 1. The system within the dashed-line is the approximate system of degree $2 n+2$ that we shall work with. This system has a scalar output, $z$, and a $2 \times 1$ vector input, $w$ with state space realization

$$
\begin{aligned}
& \dot{x}_{g}=\left[\begin{array}{cc}
0 & 1 \\
-\left(\omega_{\phi}^{2}-k_{1 \phi}\right) & -2 \zeta_{\phi} \omega_{\phi}
\end{array}\right] x_{g}+\left[\begin{array}{l}
0 \\
1
\end{array}\right] \widehat{u}=A_{\phi} x+B_{g} \widehat{u} \\
& \hat{y}=\left[\begin{array}{ll}
1 & 1
\end{array}\right] x_{g}=C_{g} x_{g}
\end{aligned}
$$

for $(s+1) G_{\phi}$, and a realization

$$
\begin{aligned}
& \dot{x}_{p}=A_{p} x_{p}+B_{p} z \\
& \hat{w}=C_{p} x_{p}
\end{aligned}
$$

for $P$. Combining these two state space models, we arrive at the following realization for the approximate system:

$$
\begin{aligned}
& \dot{x}=\left[\begin{array}{cc}
A_{\phi} & B_{g} C_{p} \\
-B_{p} \alpha_{\phi} C_{g} & A_{p}
\end{array}\right] x+\left[\begin{array}{c}
B_{g} \\
0
\end{array}\right] w=\hat{A}_{\phi} x+B w \\
& z=\left[\begin{array}{ll}
-\alpha_{\phi} C_{g} & 0
\end{array}\right] x=C_{\phi} x
\end{aligned}
$$

where $x=\left[x_{g}^{T}, \quad x_{p}^{T}\right]^{T}$. When $\phi$ is modulated according to (18), the equations (29)-(30) describe a finitedimensional linear time-varying system.

We now describe the design procedure for choosing $W_{p}$ and $T_{m}$ in (18) so as to stabilize (19). This is done in the following two steps. In the first step, we describe how $W_{p}$ and $T_{m}$ can be chosen so that (18) stabilizes (29)-(30). In the second step, we derive additional conditions that need to be satisfied by $W_{p}$ and $T_{m}$ so that (18) stabilizes the actual system in (19).

The following lemma provides the conditions for stabilization of (29)-(30) using (18). 
Lemma 1: The system in (29)-(30) with the control strategy (18) is asymptotically stable if and only if there exists a periodic (with period $T_{m}$ ), positive definite function $P$ such that

$$
\dot{P}+\hat{A}_{\phi}^{T} P+P \hat{A}_{\phi}<0
$$

for all $t \geq 0$.

The design procedure consists of choosing $W_{p}$ and $T_{m}$ so that (31) is satisfied for some $P$ and so that

$$
\phi_{\mathrm{av}}=W_{p} \phi_{1}+\left(1-W_{p}\right) \phi_{2}
$$

where $\phi_{\mathrm{av}}$ is the desired operating condition. Given a $\phi_{\mathrm{av}}$, once stable operating points $\phi_{1}$ and $\phi_{2}$ are found such that $\phi_{1}<\phi_{\mathrm{av}}<\phi_{2}$, eq.(32) determines $W_{p}$. Therefore, what remains is to show how $T_{m}$ can be chosen so that (31) is satisfied.

In order to determine $T_{m}$ from (31), fix $T_{m}$ and subdivide the time interval $\left[0, T_{m}\right]$ into $M$ equal parts, $0<\epsilon<2 \epsilon<\cdots<M \epsilon=T_{m}$. Let us denote by $\dot{P}_{k}, P_{k}, \hat{A}_{k}$ and $C_{k}$ the values of $\dot{P}, P, \hat{A}_{\phi}$ and $C_{\phi}$ respectively at time $k \epsilon$. Now, if the subdivision is suffciently fine (or if $\dot{P}$ is piecewise linear), then

$$
P_{k}=P_{0}+\frac{\epsilon}{2}\left(\dot{P}_{0}+\dot{P}_{k}+2 \sum_{j=1}^{k-1} \dot{P}_{j}\right)
$$

for $k \geq 1$. Periodicity of $P$ implies that $P_{0}=P_{M}$. This, along with (33) at $k=M$ and the periodicity of $\dot{P}$, gives

$$
\dot{P}_{0}=\dot{P}_{M}=-\sum_{k=1}^{M-1} \dot{P}_{k}
$$

Using the above developments, the linear matrix inequality (LMI) conditions in Lemma 1 can be replaced by the finite set of sufficient LMIs:

$$
\begin{gathered}
P_{0}>0 \\
P_{k}=P_{0}+\frac{\epsilon}{2}\left(\dot{P}_{0}+2 \sum_{j=1}^{k-1} \dot{P}_{j}+\dot{P}_{k}\right)>0 \\
-\left(\sum_{m=1}^{M-1} \dot{P}_{m}\right)+\hat{A}_{0}^{T} P_{0}+P_{0} \hat{A}_{0}<0 \\
\dot{P}_{k}+\hat{A}_{k}^{T} P_{k}+P_{k} \hat{A}_{k}<0
\end{gathered}
$$

where $k=1,2, \cdots, M-1$. Here, the unknowns are $\left(P_{0}, \dot{P}_{1}, \cdots, \dot{P}_{M-1}\right)$. These matrices can be computed using efficient numerical techniques that have been developed in the LMI literature [28] (for example the MATLAB LMI toolbox).

We now show that (18) stabilizes the original system (19) if $T_{m}$ is chosen so that $P$ satisfies a linear matrix inequality that is somewhat stronger than (31). Noting that the difference between the approximate 
and actual system is represented by $\Delta$, it follows that if $D$ can admit perturbations large enough compared to $\Delta$, then stabilization of the actual system is possible. This is stated more formally in the two lemmas below.

Let $\mathcal{L}_{2}$ denote the Hilbert space of functions of time with finite energy. Roughly speaking, a function $f$ is in $\mathcal{L}_{2}$ if and only if

$$
\int_{0}^{\infty} f(t)^{T} f(t) d t<\infty
$$

We can view the approximate system with input $w$ and output $z$ in Figure 1 as a mapping from $\mathcal{L}_{2}$ into $\mathcal{L}_{2}$. For each input $w$ of finite energy, the system generates an output $z$ whose energy is scaled by a factor. The largest scaling factor, obtained by searching over all finite energy inputs, is called the induced norm of the system. The lemma below gives a sufficient condition for stability of the original system and is an application of the small gain theorem [29].

Lemma 2: Let $\gamma>0$ be given, $P$ be the corresponding uniform approximation defined in (24) and the equivalence ratio $\phi$ satisfy the control law (18) for some $\left(\phi_{1}, \phi_{2}, W_{p}, T_{m}\right)$. Suppose that the induced norm of the time-varying system from $w$ to $z$ in Figure 1 is strictly less than $\gamma$. Then, the infinite dimensional system (19) with the control law (18) is stable.

The above lemma suggests that the design variables $\left(\phi_{1}, \phi_{2}, W_{p}, T_{m}\right)$ are to be selected so that the induced norm of the approximate system satisfies a specified bound. Such an induced norm can be guaranteed to exist if an LMI condition that is similar to that in (31), and somewhat stronger, is satisfied. This is stated in the following lemma.

Lemma 3: Let $\gamma>0$ be given. The approximate system (29)-(30) with the control strategy (18) has induced norm strictly less than $\gamma$ if and only if there exists a periodic (with period $T_{m}$ ) positive definite function $P$ such that

$$
\left[\begin{array}{cc}
\dot{P}+\hat{A}_{\phi}^{T} P+P \hat{A}_{\phi}+C_{\phi}^{T} C_{\phi} & P B \\
B^{T} P & -\gamma^{2} I
\end{array}\right]<0
$$

for all $t \geq 0$.

\subsubsection{The Control Design Procedure}

The summary of Lemmas 1 and 3 is the following: The switching strategy in (18) is guaranteed to stabilize the combustion instability model in (19) if a certain LMI condition is satisfied. The question that arises is if indeed this condition is satisfied for a given combustion system. In particular, given a desired operating condition $\phi_{a v}$, and values $\phi_{1}$ and $\phi_{2}$ that are such that $\phi_{1}<\phi_{\mathrm{av}}<\phi_{2}$, the problem is to determine the 
design procedure for calculating $T_{m}$ such that the LMI condition (39) can be satisfied. We address this question by considering the following three cases:

(a) Stable-Stable Switching ${ }^{2}$ : The system (17) is stable at $\phi_{1}$ and $\phi_{2}$.

(b) Stable-Unstable Switching: The system (17) is stable at one of the points (say $\phi_{1}$ ) and unstable at the other $\left(\phi_{2}\right)$.

(c) Unstable-Unstable Switching: The system (17) is unstable at $\phi_{1}$ and $\phi_{2}$.

Note that, in all of the above cases, $\phi_{1}$ and $\phi_{2}$ must satisfy $\phi_{1}<\phi_{\mathrm{av}}<\phi_{2}$ so that the time-averaged equivalence ratio can equal $\phi_{\text {av }}$, i.e. (32) holds for some $0 \leq W_{p} \leq 1$. These cases are considered in some detail below.

(a) Stable-Stable Switching: If $\phi_{1}$ and $\phi_{2}$ are stable operating points, and the goal is to switch between these two points and still ensure stability, essentially one needs to switch sufficiently slowly so that any transients that are induced due to the switching die down during the switching period. That is, if $T_{m}$ is large enough, these transients are guaranteed to die down and hence ensure stability. In fact, it can be shown that the LMI conditions in (39) hold for a sufficiently large $T_{m}$ irrespective of $W_{p}$. Since stability in this case is independent of the pulse width $W_{p}$, any desired operating equivalence ratio $\phi_{\mathrm{av}}$ between $\phi_{1}$ and $\phi_{2}$ can be achieved by simply choosing $W_{p}$ according to (32). A procedure to compute the design parameters is the following:

(1) Select $\phi_{1}$ and $\phi_{2}$ such that $\phi_{1} \leq \phi_{\mathrm{av}} \leq \phi_{2}$ and the system (17) is stable at $\phi_{1}$ and $\phi_{2}$.

(2) Choose $W_{p}$ according to (32).

(3) Choose a large period $T_{m}^{b i g}$ and perform bisection between $\left[0, T_{m}^{b i g}\right]$ until the smallest time period $T_{m}^{m i n}$ for which the LMIs in (39) hold.

Any $T_{m} \geq T_{m}^{m i n}$ will result in stability of the controlled system (17).

(b) Stable-Unstable Switching ${ }^{3}$ : Unlike case (a), "instability" or pressure amplification can result in this case either from switching or because of operating at an unstable point. Therefore, the fraction of

\footnotetext{
${ }^{2}$ This case corresponds to the behavior of the combustor considered in [2] near the operating point $\phi_{\mathrm{av}}=0.7$ with equivalence ratio modulated between two stable points.

${ }^{3}$ This case corresponds to the combustor behavior discussed in [2] near $\phi_{\mathrm{av}}=0.9$ with switching between a stable and an unstable point.
} 
time spend at the unstable operating point is relevant to stability of the overall system. That is, stability is determined by both $W_{p}$ and $T_{m}$. As a result, not all $\phi_{\mathrm{av}}$ between $\phi_{1}$ and $\phi_{2}$ can be achieved by switching.

The set of all $W_{p}$ and $T_{m}$ (equivalently, $\phi_{\mathrm{av}}$ and $T_{m}$ ) that result in stability of (17) under the control strategy (18) can be computed as follows:

(1) Select $\phi_{1}$ and $\phi_{2}$ such that $\phi_{1} \leq \phi_{\mathrm{av}} \leq \phi_{2}$ and the system (17) is stable at $\phi_{1}$ and unstable at $\phi_{2}$.

(2) Choose a period $T_{m}$. Perform bisection on $W_{p}$ between $[0,1]$ to determine the smallest $W_{p}^{\text {min }}$ for which the LMIs in (39) hold.

(3) Repeat Step (2) for a different $T_{m}$.

For any period $T_{m}>0$, the pulse width $W_{p}=1$ guarantees stability as it corresponds to operation at the stable point $\phi=\phi_{1}$. Similarly, $W_{p}=0$ results in instability. The bisection in Step (2) computes the smallest pulse width needed to guarantee stability for a fixed pulse period $T_{m}$. It is clear that large $T_{m}$ and large $W_{p}$ (or $\phi_{\text {av }}$ close to $\phi_{1}$ ) result in stability of the controlled system.

(c) Unstable-Unstable Switching: It is easy to see that large periods result in instability of the original system. Therefore, this case does not fit into the concept of stabilization via low frequency switching. That is, the system cannot be stabilized using the switching strategy in (18) if $\phi_{1}$ and $\phi_{2}$ correspond to unstable operating points, almost always.

\subsection{Non-ideal Switching}

The switching strategy given by (18) assumes that transition between the two equivalence ratios is instantaneous. But, in general, the transition time depends on the actuator bandwidth (i.e. opening and closing times of the injector valve) as well as the time taken for the reaction zone to reach the new equivalence ratio. Consider the following non-ideal switching:

$$
\phi(t)= \begin{cases}\phi_{\mathrm{av}} & \text { for } t=0 \\ \widehat{\phi}_{1}(t) & \text { for } 0 \leq t<\delta \\ \phi_{1} & \text { for } \delta \leq t<W_{p} T_{m}-\delta \\ \widehat{\phi}_{2}\left(t-W_{p} T_{m}+\delta\right) & \text { for } W_{p} T_{m}-\delta \leq t<W_{p} T_{m}+\delta \\ \phi_{2} & \text { for } W_{p} T_{m}+\delta \leq t<T_{m}-\delta \\ \widehat{\phi}_{3}\left(t-T_{m}+\delta\right) & \text { for } T_{m}-\delta \leq t<T_{m} \\ \phi_{\mathrm{av}} & \text { for } t=T_{m}\end{cases}
$$

where $\delta>0$ is the transition time, $\widehat{\phi}_{i}$ 's are continuous functions of time that describe the variation of $\phi$ during transition periods, and the remaining variables are as before. It turns out that the delay $e^{-\tau_{\phi} s}$ may 
not be a bounded operator and some assumptions are needed to extend the analysis of the previous section. We assume that the effect of non-ideal continuous switching can be approximated by that of a finite number of small ideal swicthes. In particular,

$$
\frac{e^{-\tau_{\phi} s}}{s+1} \approx \sum_{k=1}^{N} \alpha_{k \phi} \frac{e^{-\tau_{k} s}}{s+1}
$$

for some $\alpha_{k \phi}$ 's of the form in the previous section. With this assumption, it can be shown that (39) gives sufficient conditions for stability of the original system provided that the approximate model (29)-(30) is constructed with $N$ delay terms instead of two as in the previous section.

\section{Stabilization in the presence of hysteresis}

In the previous section, we implicitly assumed that the combustor parameter vector $f(\phi)=\left(\zeta_{\phi}, \omega_{\phi}, k_{1 \phi}, k_{2 \phi}, \tau_{\phi}\right)$ is single-valued at each $\phi$. As noted in [5], often a hysteresis mechanism is present in combustion systems. This in turn implies that $f(\phi)$ is multi-valued between $\phi_{1}$ and $\phi_{2}$. Interestingly enough, even in this case, stabilization is possible using the same switching strategy as in (40), with the underlying conditions involving functional forms of $f(\phi)$ for both branches of hysteresis (Figure 2). To do this, consider the hysteresis cycle shown in Figure 2 and suppose that the desired operating point is $\phi_{\mathrm{av}}$. Let us denote the lower and upper halves of hysteresis cycle by $f_{1}$ and $f_{2}$ respectively. Also, suppose that $\delta$ is the transition time between $\phi_{1}$ and $\phi_{\mathrm{av}}$ as well as between $\phi_{\mathrm{av}}$ and $\phi_{2}$. Thus, in the presence of hysteresis, switching between $\phi_{1}$ and $\phi_{2}$ leads to combustor parameter variation given by:

$$
f(\phi)(t)= \begin{cases}f_{1}\left(\phi_{\mathrm{av}}\right) & \text { for } t=0 \\ f_{1}\left(\widehat{\phi}_{1}(t)\right) & \text { for } 0 \leq t<\delta \\ f_{1}\left(\phi_{1}\right)=f_{2}\left(\phi_{1}\right) & \text { for } \delta \leq t<W_{p} T_{m}-\delta \\ f_{2}\left(\widehat{\phi}_{2}\left(t-W_{p} T_{m}+\delta\right)\right) & \text { for } W_{p} T_{m}-\delta \leq t<W_{p} T_{m}+\delta \\ f_{2}\left(\phi_{2}\right)=f_{1}\left(\phi_{2}\right) & \text { for } W_{p} T_{m}+\delta \leq t<T_{m}-\delta \\ f_{1}\left(\widehat{\phi}_{3}\left(t-T_{m}+\delta\right)\right) & \text { for } T_{m}-\delta \leq t<T_{m} \\ f_{1}\left(\phi_{\mathrm{av}}\right) & \text { for } t=T_{m}\end{cases}
$$

which is no more general than the parameter variations generated by the non-ideal switching strategy (40). Therefore, (39) once again corresponds to sufficient conditions for stabilization with the the approximate system represented using $N$ delays rather than two, as discussed in the previous section.

Let us examine some of the consequences of our analytical results for stabilization in the presence of hysteresis. First, there is a minimum value of pulse period $T_{m}$ below which stabilization is not possible. Intuitively, this is because rapid switching results in very little time for transients to die out. Second, since part of the hysteresis cycle leads to unstable operating condition, pulse width cannot be arbitrary for stabilization. This is similar to the stable-unstable switching we considered. A third statement we can make is 


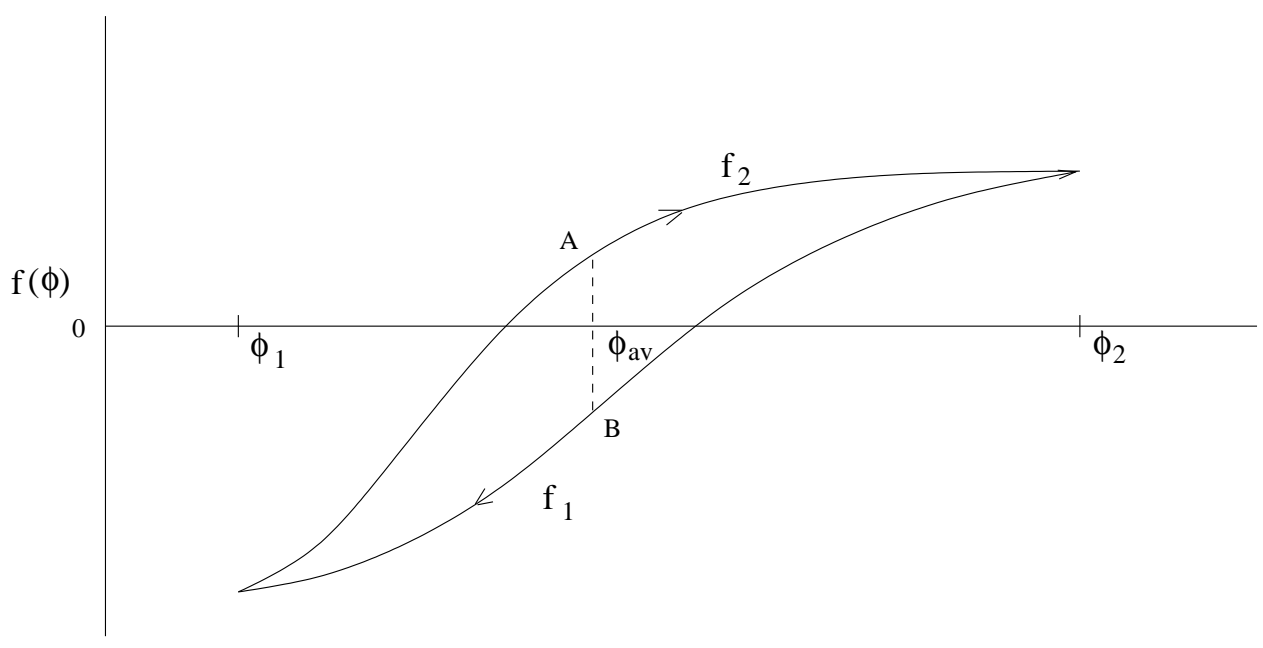

Figure 2: Hysteresis curve

that due to the non-zero transition time $\delta$, short pulses may not be stabilizing. These statements corroborate the following observations made in [5]:

(O1) Pulsing at a slower frequency than the unstable frequency stabilizes the system.

(O2) Shorter pulses tend to be less effective, in general.

Recall that $\phi_{1}$ and $\phi_{2}$ are design variables that must be chosen along with $W_{p}$ and $T_{m}$ so that equation (32) and the LMIs (35)-(38) hold to guarantee stability. In the presence of hysteresis, an additional condition which we implicitly assumed must hold. This condition is the following: $\phi_{1}$ and $\phi_{2}$ must be sufficiently apart so that the lower and upper halves of the hysteresis cycle meet at $\phi_{1}$ and $\phi_{2}$ :

$$
f_{1}\left(\phi_{1}\right)=f_{2}\left(\phi_{1}\right) \text { and } f_{1}\left(\phi_{2}\right)=f_{2}\left(\phi_{2}\right)
$$

In other words, the flow rate of the fuel injector should be large enough so that we can switch between $\phi_{1}$ and $\phi_{2}$ satisfying the above condition. This corroborates yet another observation made in [5]:

(O3) The flow rate needed to accomplish successful pressure suppression is substantial (about $25 \%$ of the primary fuel-flow rate).

It is quite likely that the primary loop of the hysteresis curve in $[5,4]$ corresponded to an equivalence ratio change that is commensurate with a $25 \%$ change in the fuel-flow rate. This discussion shows that (39) provides sufficient conditions for stabilization with infinite switching in the presence of hysteresis.

When hysteresis is present, a considerably simpler control strategy suffices: a single pulse of sufficient duration and height. This can be explained using Figure 2. Let us assume that operation begins on the 
upper half of the hysteresis cycle at an equivalence ratio of $\phi_{\mathrm{av}}$ (point A in Figure 2). For stabilization using hysteresis, we need a pulse of sufficient duration and height so that the equivalence ratio $\phi_{2}$ can become effective following which the system can return to the desired operating point B in Figure 2 along the lower branch during the off-period. Thus, during this single period, the switching strategy takes the equivalence ratio from $\phi_{\mathrm{av}}$ to $\phi_{2}$ and back. As long as the pulse duration and width are large enough to allow transition from $\phi_{\text {av }}$ to $\phi_{2}$, stabilization will occur. This confirms the observations (O1)-(O3) in [5]. As with the infinite switching strategy, we note that the robustness of this single pulse strategy is non-existent; as long as perturbations are present, the operating point will move from $B$ to $A$ causing pulsing to be invoked repeatedly.

We note that this paper does not answer the question regarding how general the existence of hysteresis is in combustors or what the responsible mechanisms are in a combustion system for the hysteresis. However, once it is detected, this paper gives guidelines for determining the number, width, and height of secondaryfuel pulses to efficiently stabilize the system.

\section{Simulations}

We now present numerical results to show that a combustion rig, modeled by (17), can be stabilized using an open-loop switching strategy with a nonzero transition time. The simulation model is:

$$
\ddot{\eta}+2 \zeta_{\phi} \omega_{\phi} \dot{\eta}+\left(\omega_{\phi}^{2}-k_{1 \phi}\right) \eta+k_{2 \phi} \eta\left(t-\tau_{\phi}\right)=w
$$

where $w$ is a process noise vector. We have added $w$ to the system dynamics to represent the effects of unmodeled dynamics and external disturbances. This addition does not affect stability properties of the system. Numerical results are given for the stable-stable and stable-unstable switching cases corresponding to the experimental conditions in [2]. Our results with the linear simulation model corroborate those of [2].

For the numerical results presented below, we used an unstable frequency $\omega$ of $300 \mathrm{~Hz}$, and an equivalence ratio transition time $\delta$ of $0.0125 \mathrm{sec}$ as in [2]. White noise with a covariance of 200 was used as the process noise $w$. Initial conditions were randomly set; but the same initial state was used for simulation at desired operating point $\phi_{\text {av }}$ with no switching, and simulation with open-loop switching strategy. Table 1 gives numerical values of other model parameters. These parameters were used to compute $W_{p}$ using (32)

and the smallest period $T_{m}^{m i n}$ using the LMIs in (39), both in the stable-stable and stable-unstable switching case, and are as follows.

Stable-Stable Non-ideal Switching: $W_{p}=0.5$ and $T_{m}^{\min }=0.05$ 
Stable-Unstable Non-ideal Switching: $W_{p}=0.5$ and $T_{m}^{\min }=0.05$

For solving the LMIs, we used $\epsilon=\delta / 2$.

\begin{tabular}{||c|c|c||}
\hline \hline Parameter & Stable-Stable & Stable-Unstable \\
\hline$\left(\phi_{1}, \phi_{\mathrm{av}}, \phi_{2}\right)$ & $(0.65,0.7,0.75)$ & $(0.8,0.9,1)$ \\
\hline$\left(\omega_{\phi_{1}}, \omega_{\phi_{\mathrm{av}}}, \omega_{\phi_{2}}\right)$ & $(300,300,300)$ & $(300,300,300)$ \\
\hline$\left(\zeta_{\phi_{1}}, \zeta_{\phi_{\mathrm{av}}}, \zeta_{\phi_{2}}\right)$ & $(0.05,0,0.01)$ & $(0.05,0,-0.005)$ \\
\hline$\left(k_{1 \phi_{1}}, k_{1 \phi_{\mathrm{av}}}, k_{1 \phi_{2}}\right)$ & $\left(\omega_{\phi_{1}}, \omega_{\phi_{\mathrm{av}}}, \omega_{\phi_{2}}\right)$ & $\left(\omega_{\phi_{1}}, \omega_{\phi_{\mathrm{av}}}, \omega_{\phi_{2}}\right)$ \\
\hline$\left(k_{2 \phi_{1}}, k_{2 \phi_{\mathrm{av}}}, k_{2 \phi_{2}}\right)$ & $\left(\omega_{\phi_{1}}, \omega_{\phi_{\mathrm{av}}}^{2}, \omega_{\phi_{2}}\right)$ & $\left(\omega_{\phi_{1}}, \omega_{\phi_{\mathrm{av}}}^{2}, \omega_{\phi_{2}}\right)$ \\
\hline$\left(\tau_{\phi_{1}}, \tau_{\phi_{\mathrm{av}}}, \tau_{\phi_{2}}\right)$ & $\left(\pi / \omega_{\phi_{1}}, 2 \pi / \omega_{\phi_{\mathrm{av}}}, \pi / \omega_{\phi_{2}}\right)$ & $\left(\pi / \omega_{\phi_{1}}, 2 \pi / \omega_{\phi_{\mathrm{av}}}, \pi / \omega_{\phi_{2}}\right)$ \\
\hline$\left(W_{p_{m}}, T_{m}\right)$ & $(0.5,0.2)$ & $(0.5,0.2)$ \\
\hline \hline
\end{tabular}

Table 1: Numerical values of parameters used in simulations. Equivalence ratios $\phi_{1}, \phi_{\mathrm{av}}, \phi_{2}$, pulse width $W_{p}$ and period $T_{m}$ are from [2].

\subsection{Simulation 1: Stable-Stable Switching}

Here, the desired operating condition is unstable and the strategy is to switch between two stable points "hopping over" the instability. This case corresponds to experimental results near the operating point $\phi_{\mathrm{av}}=$ 0.7 (at an inlet temperature of $367 \mathrm{~K}$ ) presented in [2]. Numerical values used for simulations are given in Table 1.

Figure 3 shows pressure response of the simulation model (42). The left half of the plot shows pressure response at the desired equivalence ratio $\phi_{\mathrm{av}}$ with no switching. The right half of the plot shows reduction in pressure obtained with open-loop switching. The plot at the bottom shows switching sequence with nonzero transition time. It is clear that the open-loop switching strategy is stabilizing. This figure compares very well with the experimental results presented in [2] (see Figure 10a of [2]).

\subsection{Simulation 2: Stable-Unstable Switching}

In this case, the desired operating condition is unstable and is not an isolated instability as in the previous case that allowed us to hop over to a stable operating point. This condition corresponds to the experimental results near the operating point $\phi_{\mathrm{av}}=0.9$ given in [2] and the open-loop control strategy is to switch between a stable operating point and an unstable operating point.

Figure 4 shows pressure response of the linear simulation model (42). The left half of the plot shows pressure response at the desired equivalence ratio $\phi_{\mathrm{av}}$ with no switching. The right half of the plot shows the 
effect of open-loop switching. The large jumps in amplitude is caused by switching to an unstable operating point. The plot at the bottom shows switching sequence with nonzero transition time. As in the previous case, this figure compares very well with the experimental results presented in [2] (see Figure 7a of [2]).

\subsection{Simulation 3: Evaluation of Results in [1]}

The approach in [1] is somewhat different from that in [2] in that the open-loop control strategy is evaluated at three different operating points. The switching period is still chosen to be considerably smaller than the unstable frequency. Since the control strategy evaluated in this paper consists of slow switching, we discuss the conditions cited in section 3 and their ability to predict the results of [1] as well, in this section.

The minimum and maximum overall equivalence ratios used in [1] are $\phi_{1}=0.73$ (pulse injector off) and $\phi_{2}=0.85$ (pulse injector on). This means that, unlike the previous cases, we can attain any desired equivalence ratio $\phi_{\mathrm{av}}$ in the range $\left[\phi_{1}, \phi_{2}\right]$ by selecting an appropriate pulse width $W_{p}$ according to (32). The experimental results given in [1] correspond to: (i) $W_{p}=0, \phi_{\mathrm{av}}=0.73$ (fuel injector off), (ii) $W_{p}=0.2$, $\phi_{\mathrm{av}}=0.826$ (with a duty-cycle of $80 \%$ ), and (iii) $W_{p}=0.64, \phi_{\mathrm{av}}=0.7732$ (with a duty-cycle of $36 \%$ ). Results in [1] at a switching frequency of $50 \mathrm{~Hz}\left(T_{m}=0.02 \mathrm{sec}\right)$ show that pressure oscillations are slightly larger with the switching strategy (ii) is applied than when the injector is off (as in (i)). It is also shown in [1] that pressure oscillations are significantly reduced by applying the strategy (iii). These observations can be explained with the same analytical developments of Section 3 with a suitable choice of the combustor model parameters:

The following values are chosen for numerical simulation:

$$
\begin{gathered}
\omega_{\phi}=600 \pi=k_{1 \phi}=k_{2 \phi}=\pi / \tau \\
\zeta_{\phi_{1}}=0.01, \zeta_{0.7732}=0.05, \zeta_{0.826}=0.005, \zeta_{\phi_{2}}=0.001
\end{gathered}
$$

These choices are motivated by the experimental conditions in [1].A transition time of $\delta=0.005 \mathrm{sec}$ was used. The corresponding results shown in Figure 5. Indeed stabilization occurs in case (iii) and larger oscillations are present in case (ii). This is similar to the observations in Figures 5,7, and 8 in [1].

\section{Discussion}

The discussions in Sections 2-5 show that an analytical model can be derived that provides explanations and guidelines for the success of an open-loop control strategy with a large enough switching period. It was 
shown that an appropriate switching strategy can be found for stabilizing the combustor both while switching between two stable operating points and while switching between a stable and an unstable operating points. It was also shown that the numerical simulation studies match these analytical predictions and more importantly, match the experimental observations in $[1,2]$.

The discussions in section 3 showed that the success of a slowly switching open-loop control strategy essentially depends on the nature of the parameter vector $f(\phi)$ defined in (20). If $f(\phi)$ belongs to one of the cases (a)-(c) defined in section 3.1.2, then $f(\phi)$ determines the feasibility of the open loop strategy with the specific case fixing the parameters of the switching scheme (18). Therefore, a determination of how $f(\phi)$ varies with $\phi$ in a given rig is useful for determining the control strategy. Of the parameters $\left(\omega_{\phi}, \zeta_{\phi}, k_{1 \phi}, \alpha_{1 \phi}, \alpha_{2 \phi}, \tau_{\phi}\right)$ in $f(\phi)$, the most sensitive is $\tau$. As shown in Eq. (14), in the case when the instability mechanism is due to variations in $u^{\prime}, \tau=\tau_{f} ; \tau_{f}$ changes with the burning velocity $S_{u}$, which in turn is quite sensitive to changes in $\phi$. For example, in propane-air mixture, a $25 \%$ change in $\phi$ leads to a $400 \%$ change in $S_{u}$ [30]. Also, noting the structure of Eq. (19), it is clear that changes in the time-delay lead to a more drastic change in the stability pattern of the underlying system rather than changes in the other parameters of $f(\phi)$. In particular, suppose we are interested in operating conditions $\phi_{1}$ and $\phi_{2}$ both of which are stable, and $\phi_{a v}$ is unstable. If the dominant effect of $\phi$ is on $\tau$, then the system should exhibit a stableunstable-stable pattern as $\tau$ increases from a value that corresponds to $\phi_{1}$, through $\phi_{a v}$ to $\phi_{2}$. Such stability "bands" are known to exist in time-delay systems in general, and in the experimental rig in [2] in particular (Figure 6). It is worth noting that in [2], care is taken to ensure that the equivalence ratio fluctuations in the feed-system are decoupled from the burning zone. It is therefore quite likely that the configuration in [2] consists of an instability mechanism where fluctuations in $u^{\prime}$ are dominant. The above discussions indicate that the results presented in this paper corroborate the observations in [2] rather closely. First, the instability properties of the uncontrolled process parallel those of the model in (13), Second, a slow switching strategy as in [2] has been shown in section 3 to lead to stability properties that are quite similar to the experimental observations in [2].

We have also discussed the results in [1] in section 3. It should be noted that the combustor in [1] differs from that in [2] in two respects. First, variations in $\phi^{\prime}$ are present in the rig in [1], contrary to the case in [2] where a decoupler is introduced between the feed system and the burning zone. This implies that the time-delay in [1] may be due to $\tau_{c}$. Second, the control strategy in [1] was attempted at different duty-cycles; this is in contrast to that in [2] where the duty-cycle was fixed at $50 \%$ and only the values $\phi_{1}$ and $\phi_{2}$ between which $\phi$ was changed were altered. As mentioned in sections 2 and 5, if the duty cycle is changed, the operating point, $\phi_{\mathrm{av}}$, shifts. The success of the switching strategy in such a case is simply governed by the 
value of $f\left(\phi_{\mathrm{av}}\right)$. This is quite distinct from the strategy in [2] where $\phi_{\mathrm{av}}$ is fixed. In fact, it can be argued that in this case, to achieve stabilization, one does not need to switch between two values in a periodic manner but could simply operate at a $\phi_{\mathrm{av}}$, based on the value $f\left(\phi_{\mathrm{av}}\right)$. If $f\left(\phi_{\mathrm{av}}\right)$ corresponds to a stable value, slow switching around this operating condition will still lead to stability, whereas switching around an unstable value of $f\left(\phi_{\mathrm{av}}\right)$ leads to a more unstable response, which may have been the cause of the observations in Figures 7 and 8 in [1].

Yet another question that is raised in [2] is the number and location of multiple fuel-injectors required to ensure the success of the proposed switching strategy. Again, this can be quantified using our model and $f(\phi)$. The ultimate goal is the realization of two values $\phi_{1}^{*}$ and $\phi_{2}^{*}$ around the desired operating point $\phi_{\mathrm{av}}$ at which stability can be realized. Noting that these values correspond to the equivalence ratio at the burning section, in the situation where multiple fuel-injectors are used, the problem is to identify at least two choices among various sequences of pulses of fuel-injector that correspond to these desired values. Suppose that there are $n$ fuel-injectors, located at $n$ different locations, and each can, at any instant, be in the on- or off-mode. The sequence of pulses from these $n$ injectors at a time instant can be expressed as $\left\{e_{1}, \ldots, e_{n}\right\}$ where each $e_{i}$ can take a value 1 or 0 depending on whether the $i$ th injector is in the on- or off-mode, Hence, it follows that $2^{n}$ possible sequences $S_{i}$ can be generated using these $n$ fuel-injectors. The issue is therefore to identify the mapping from $S_{i}$ to $\phi_{i}$, and find the $S_{i}$ 's that correspond to the desired $\phi_{1}^{*}$ and $\phi_{2}^{*}$. In general, these sequences are bound to depend in a complex way on the configuration and boundary conditions in a given rig. Once these sequences are identified, they determine in turn when a given injector is fired. Given the quasi-static nature of the switching strategy (that is, the switching frequency is significantly smaller than the unstable frequency), the time-delay between the firing instants of any two injectors may not be a relevant parameter as was speculated in [21].

We have also showed in this paper an explanation for when and how a combustor can be stabilized using a switching strategy in the presence of hysteresis as in $[4,5]$. In particular, we have shown that the same proposition that explained the idea behind stabilization using the switching strategy in $[1,2]$ provides the analytical basis for the results in $[4,5]$ as well. We showed that our observations based on the model and the proposition matches the experimental observations in $[4,5]$.

A point worth making is about properties of open-loop control strategies in general. Unlike closed-loop control strategies, open-loop control strategies are quite vulnerable to changes in the operating parameters. As the above discussion indicated, the stability of the open-loop strategy is closely tied to $f(\phi)$, and hence its stability robustness is conditioned on the sensitivity of $f(\phi)$ to various perturbations and changes in the operating conditions. This should be kept in mind while implementing open-loop control strategies. It 
should be noted that for the switching strategy in (18), stability depends only on $f\left(\phi_{1}\right)$ and $f\left(\phi_{2}\right)$, and not on how the function $f$ behaves at other values of $\phi$.

Finally, we make a comment about the model in (17), which is a second-order, lumped, time-delay model. Obviously, a fair amount of simplification has been made in collapsing the complex dynamic interactions between acoustic modes, heat release dynamics from a distributed flame zone, fuel-injection, and mixing dynamics into a simple form. However, the description of the open-loop control strategy and its impact on the rig using the time-delay model as in (17) represents a very useful guideline and an important beginning for how the physical model can be expanded appropriately to encompass various dynamics exhibited by a combustion rig with and without active control.

\section{Acknowledgements}

This work is sponsored in part by the National Science Foundation, contract no. ECS 9713415, and in part by the Office of Naval Research, contract no. N00014-99-1-0448.

\section{References}

[1] G.A.Richards, M.J. Yip, E. Robey, L. Cowell, and D. Rawlins. Combustion oscillation control by cyclic fuel injection. Journal of Engineering for Gas Turbines and Power, 119:1-4, 1997.

[2] G.A.Richards, M.C. Janus, E. Robey, L. Cowell, and D. Rawlins. Control of flame oscillations with equivalence ratio modulation. AIAA Journal of Propulsion and Power, 15:232-240, 1999.

[3] J. Brouwer, B.A. Ault, J.E. Bobrow, and G.S. Samuelsen. "Active control for gas turbine combustors". In Twenty-third Symposium (International) on Combustion, Pittsburgh, PA, 1990. The Combustion Institute.

[4] P. Knoop, F.E.C. Culick, and E.E. Zukoski. Extension of the stability of motions in a combustion chamber by nonlinear active control based on hysteresis. Combustion Science and Technology, 123:363-376, 1997.

[5] G. Isella, C. Seywert, F.E.C. Culick, and E.E. Zukoski. A further note on active control of combustion instabilities based on hysteresis. Combustion Science and Technology, 126:381-388, 1997.

[6] M.A. Heckl. "Active control of the noise from a Rijke tube". In G. Comte-Bellot and J.E. Flowers Williams, editors, Aero- and Hydro-Acoustics, pages 211-216. Springer, Berlin Heidelberg, Berlin Heidelberg, 1986.

[7] W. Lang, T. Poinsot, and S. Candel. Active control of combustion instability. Combustion and Flame, 70:281289, 1987.

[8] G.J. Bloxsidge, A.P. Dowling, N. Hooper, and P.J. Langhorne. "Active control of an acoustically driven combustion instability". Journal of Theoretical and Applied Mechanics, supplement to vol. 6, 1987.

[9] T. Poinsot, F. Bourienne, S. Candel, and E. Esposito. "Suppression of combustion instabilities by active control". Journal of Propulsion and Power, 5(1):14-20, 1989.

[10] P.J. Langhorne, A.P. Dowling, and N. Hooper. Practical active control system for combustion oscillations. Journal of Propulsion and Power, 6(3):324-333, 1990.

[11] A. Gulati and R. Mani. Active control of unsteady combustion-induced oscillations. Journal of Propulsion and Power, 8(5):1109-1115, 1992. 
[12] K.R. McManus, U. Vandsburger, and C.T. Bowman. "Combustor performance enhancement through direct shear layer excitation". Combustion and Flame, 82:75-92, 1990.

[13] E. Gutmark, T.P. Parr, K.J. Wilson, D.M. Hanson-Parr, and K.C. Schadow. "Closed-loop control in a flame and a dump combustor". IEEE Control Systems, 13:73-78, April 1993.

[14] A.M. Annaswamy, M. Fleifil, J.P. Hathout, and A.F. Ghoniem. "Impact of linear coupling on the design of active controllers for thermoacoustic instability". Combustion Science and Technology, 128:131-180, December 1997.

[15] M. Fleifil, A.M. Annaswamy, J.P. Hathout, and A.F. Ghoniem. "The origin of secondary peaks with active control of thermoacoustic instability". In Proceedings of the AIAA Joint Propulsion Conference, Seattle, WA;Combustion Science and Technology (to appear), July 1997.

[16] J.P. Hathout, A.M. Annaswamy, M. Fleifil, and A.F. Ghoniem. "A model-based active control design for thermoacoustic instability". Combustion, Science, and Technology, 132:99-138, May 1998.

[17] A.M. Annaswamy, O. El-Rifai, M. Fleifil, and A.F. Ghoniem. "A model-based self-tuning controller for thermoacoustic instability". Combustion, Science, and Technology, 135:213-240, August 1998.

[18] Y. Neumeier and B.T. Zinn. Active control of combustion instabilities using real time identification of unstable combustor modes. In Proceedings of the IEEE Conference on Control Applications, pages 691-698, Albany, NY, 1995.

[19] J.E. Tierno and J.C. Doyle. "Multimode active stabilization of a Rijke tube". In DSC-Vol. 38. ASME Winter Annual Meeting, 1992.

[20] S. Murugappan, S. Park, A.M. Acharya, S. andAnnaswamy, E. Gutmark, and A.F. Ghoniem. Optimal control of swirl-stabilized combustor using a system identification based model. In Turbine 2000, Cesme, Turkey, August 2000.

[21] G.A. Richards. Methods to control combustion dynamics. In AGTSR Workshop, Blacksburg, VA, 1999.

[22] H.M. Najm and A.F. Ghoniem. "Modeling pulsating combustion in vortex stabilized pre-mixed flames". Combustion Sci. Tech., 94:259-278, 1993.

[23] M. Fleifil, A.M. Annaswamy, Z. Ghoniem, and A.F. Ghoniem. "Response of a laminar premixed flame to flow oscillations: A kinematic model and thermoacoustic instability result". Combustion and Flame, 106:487-510, 1996.

[24] J. P. Hathout, M. Fleifil, A. M. Annaswamy, and A. F. Ghoniem. A reduced-order model of time-delay induced combustion instability. Technical Report 2003, Massachusetts Institute of Technology, April 2000.

[25] A.P. Dowling and J.E. Ffowcs-Williams Sound and Sources of Sound. Ellis Horwood Limited, West Sussex, PO191EB, England, 1983.

[26] Vigor Yang and R. Anderson (Eds.). Liquid rocket engine combustion instability. Progress in Astronautics and Aeronautics. AIAA, 1995.

[27] A.A. Peracchio and W. Proscia. Nonlinear heat release/acoustic model for thermoacoustic instability in lean premixed combustors. In ASME Gas Turbine and Aerospace Congress, Sweden, 1998.

[28] S.P. Boyd, L. El Ghaoui, E. Feron, and V. Balakrishnan. Linear Matrix Inequalities in Systems and Control Theory. SIAM, Philadelphia, PA, 1994.

[29] C.A. Desoer and M. Vidyasagar. Feedback Systems: Input-Output Properties. Academic Press, New York, 1975.

[30] F.A. Williams. Combustion Theory. Addison-Wesley Co., Reading, MA, 1965.

[31] S. I. Niculescu, A.M. Annaswamy, J.P. Hathout, and A.F. Ghoniem. Control of time-delay induced instabilities in combustion systems. In IEEE, CDC conference (in review), 2000.

[32] T. Lieuwen and B.T. Zinn. "The role of equivalence ratio oscillations in driving combustion instabilities in low N0 $0_{x}$ gas turbines". The Twenty Seventh International Symposium on Combustion, pages 1809-1816, 1998. 


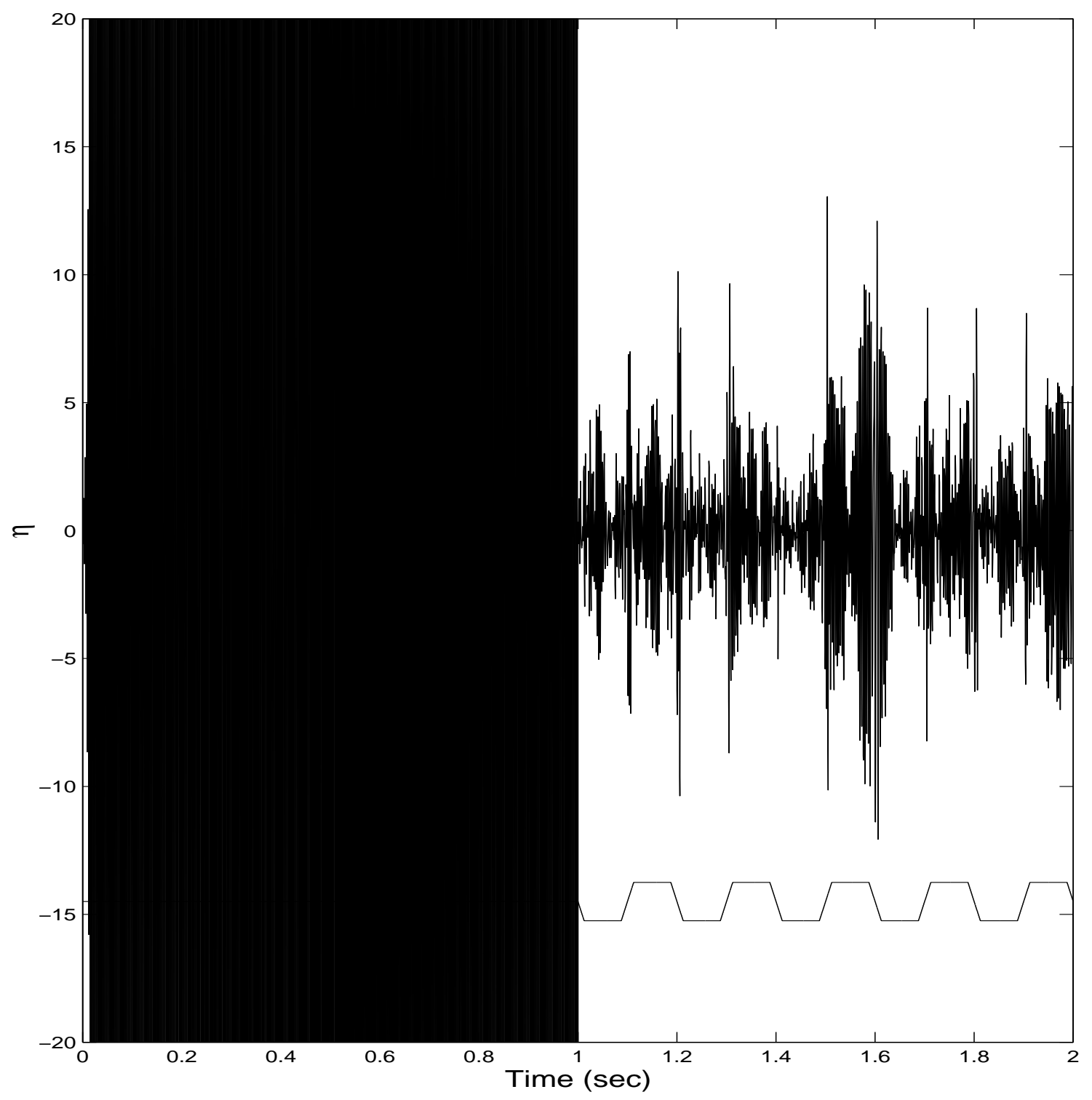

Figure 3: Stabilizing effect of open-loop equivalence ratio modulation between two stable points. Response shown on the left half corresponds to operation at $\phi_{\text {av }}$; the right half shows reduction in pressure oscillations obtained from equivalence ratio modulation. Compare with Figure 10a of [2]. 


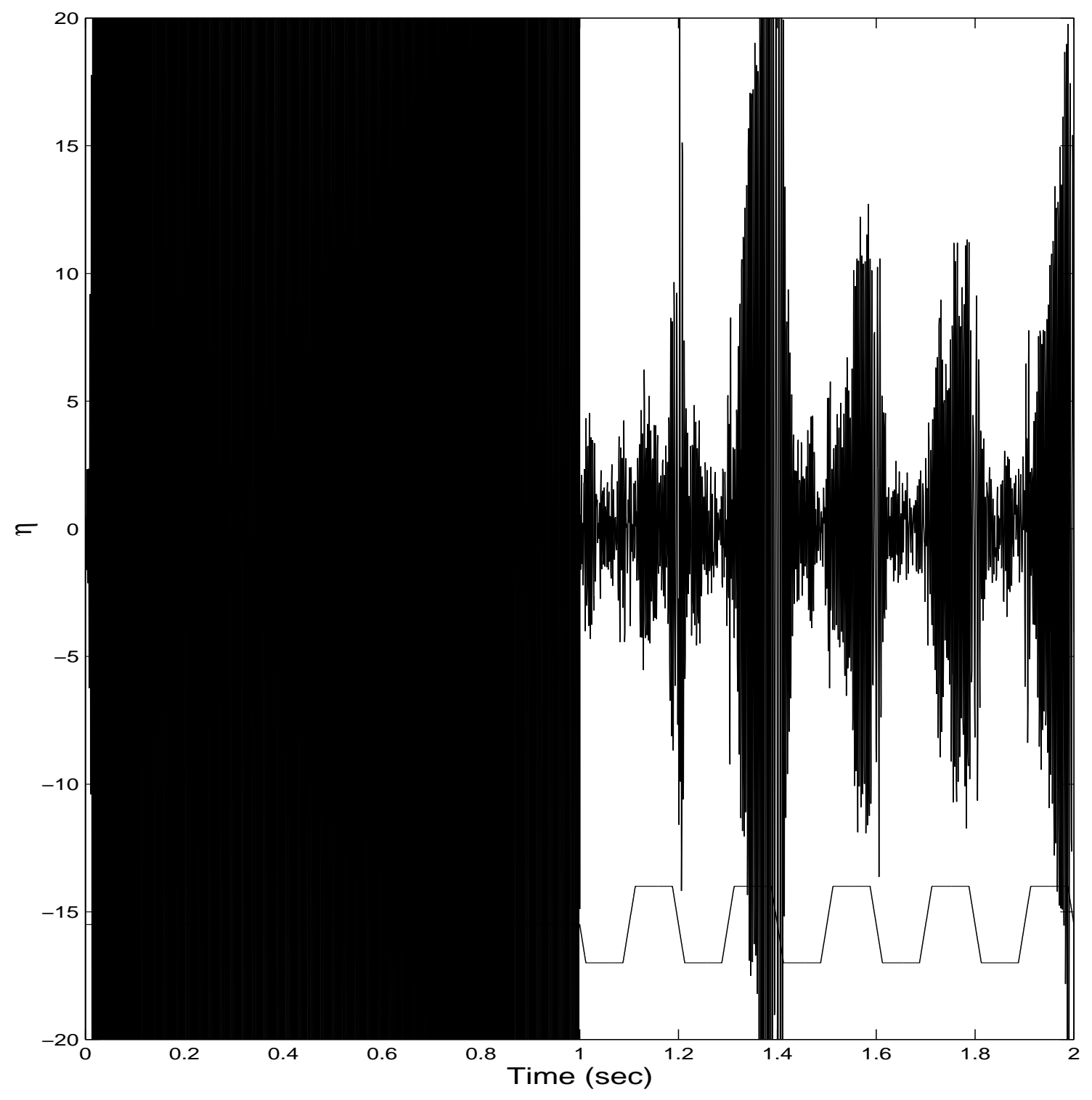

Figure 4: Stabilizing effect of open-loop equivalence ratio modulation between a stable and an unstable point. Response shown on the left half corresponds to operation at $\phi_{\mathrm{av}}$; the right half shows response with equivalence ratio modulation. Compare with Figure 7a of [2]. 

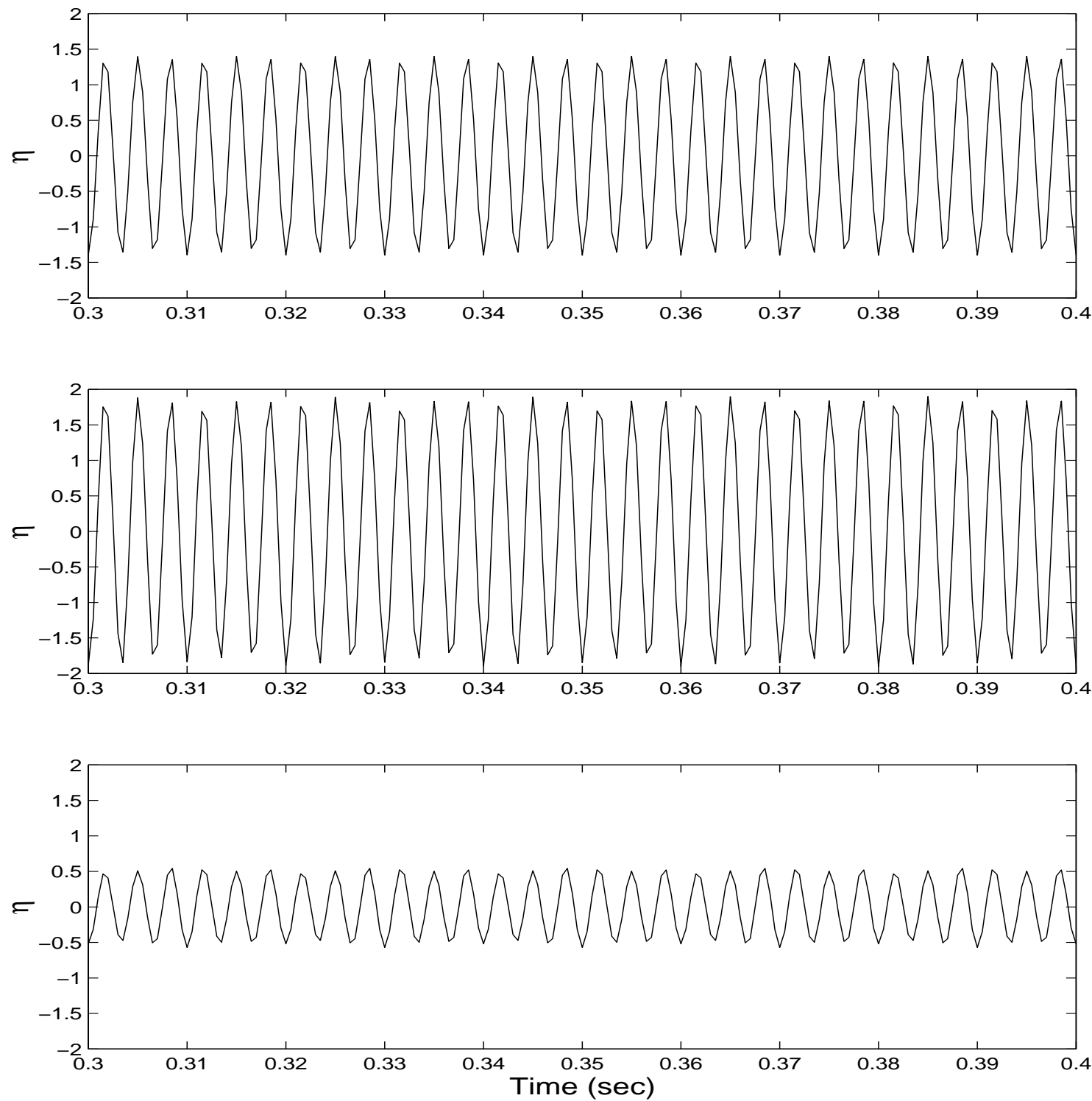

Figure 5: Response of the linear simulation model. The top plot is for operation at $\phi_{\mathrm{av}}=\phi_{1}=0.73$ with no switching; the middle plot is for operation at $\phi_{\mathrm{av}}=0.826$ with switching and a duty-cycle of $80 \%$; the bottom plot is for operation at $\phi_{\mathrm{av}}=0.7732$ with switching and a duty-cycle of $36 \%$. Compare with Figures $5,7,8$ of [1]. 